\title{
ON THE POINTWISE CONVERGENCE OF A CLASS OF NONHARMONIC FOURIER SERIES ${ }^{1}$
}

\author{
ROBERT M. YOUNG
}

\begin{abstract}
Extending a classical theorem of Levinson [1. Theorem XVIII], we show that when the numbers $\left\{\lambda_{n}\right\}$ are given by $\lambda_{n}=n+\frac{1}{4}(n>0) . \lambda_{0}=0$. and $\lambda_{-n}=$ $-\lambda_{n}(n>0)$, each function $f$ in $L^{2}(-\pi, \pi)$ has a unique nonharmonic Fourier expansion $f(t) \sim \sum_{-\infty}^{\infty} c_{n} e^{i \lambda_{n} t}$. which is equiconvergent with its ordinary Fourier series, uniformly on each closed subinterval of $(-\pi, \pi)$.
\end{abstract}

1. Introduction. A system of complex exponentials $\left\{e^{i \lambda_{n} t}\right\}$ is said to be "equivalent" to the trigonometric system $\left\{e^{i n t}\right\}$ in $L^{2}(-\pi, \pi)$-or a Riesz basis - if the mapping $e^{i n t} \rightarrow e^{i \lambda_{n} t}(-\infty<n<\infty)$ can be extended to an isomorphism on all of $L^{2}(-\pi, \pi)$. If this is the case, then each function $f$ in $L^{2}(-\pi, \pi)$ will have a unique nonharmonic Fourier expansion

$$
\left.f(t)=\sum_{-\infty}^{\infty} c_{n} e^{i \lambda_{n} t} \quad \text { (in the mean }\right)
$$

with $\left\{c_{n}\right\}$ in $l^{2}$.

The study of nonharmonic Fourier series was initiated by Paley and Wiener who showed that the system $\left\{e^{i \lambda_{n} t}\right\}$ is a Riesz basis for $L^{2}(-\pi, \pi)$ whenever each $\lambda_{n}$ is real and $\left|\lambda_{n}-n\right| \leqslant L<1 / \pi^{2}$. Ultimately, Kadec showed that the constant $1 / \pi^{2}$ could be replaced by $\frac{1}{4}$. That $\frac{1}{4}$ is in fact the "best possible" constant follows from the fact that the system $\left\{e^{ \pm i(n-1 / 4) t}: n=1,2,3, \ldots\right\}$ is already complete in $L^{2}(-\pi, \pi)$. (For a comprehensive introduction to the theory of nonharmonic Fourier series, including proofs of these assertions, see [3].)

At present it is unknown whether there exists a basis of complex exponentials for $L^{2}(-\pi, \pi)$ that is not a Riesz basis. A promising candidate is the system $\left\{e^{i \lambda_{n} t}\right\}$ with

$$
\lambda_{n}= \begin{cases}n+1 / 4, & n>0, \\ 0, & n=0, \\ n-1 / 4, & n<0\end{cases}
$$

This sequence is complete, possesses a complete and bounded biorthogonal sequence, and yet fails to be a Riesz basis (see e.g., [ 2 and 4$]$ ). The following theorem and its corollary add further evidence to support the conjecture that the aforementioned system is a basis for $L^{2}(-\pi, \pi)$.

Received by the editors September 20, 1982.

1980 Mathematics Subject Classification. Primary 42C30; Secondary 30D99.

Kev words and phrases. Biorthogonal system, equiconvergence, nonharmonic Fourier series.

1 The author is grateful to Ray Redheffer for his many helpful ideas. 
THEOREM. If the $\lambda_{n}$ are given by (1), then each function $f$ in $L^{2}(-\pi, \pi)$ has a unique nonharmonic Fourier expansion

$$
f(t) \sim \sum_{-\infty}^{\infty} c_{n} e^{i \lambda_{n} t}
$$

which is equiconvergent with its ordinary Fourier series uniformly on each closed subinterval of $(-\pi, \pi)$.

(Recall that two series $\Sigma A_{n}$ and $\Sigma B_{n}$ are equiconvergent if their difference $\Sigma\left(A_{n}-B_{n}\right)$ converges and has the sum zero.)

Since the Fourier series of an $L^{2}$ function converges to the function pointwise almost everywhere, the same must be true for the nonharmonic series.

COROLLARY. Under the hypotheses of the theorem,

$$
f(t)=\sum_{-\infty}^{\infty} c_{n} e^{i \lambda_{n} t}
$$

almost everywhere on $(-\pi, \pi)$.

The Theorem above (and more) was established by Levinson [1, Theorem XVIII] under the assumption that $\left|\lambda_{n}-n\right| \leqslant L<\frac{1}{4}$. It will be shown that with suitable modifications, together with a few special estimates, Levinson's proof carries over virtually intact.

2. Preliminary results. We shall establish several lemmas concerning the function

$$
G(z)=\left(z-\lambda_{0}\right) \prod_{n=1}^{\infty}\left(1-\frac{z}{\lambda_{n}}\right)\left(1-\frac{z}{\lambda_{-n}}\right),
$$

where the $\lambda_{n}$ are real and $\left|\lambda_{n}-n\right| \leqslant \frac{1}{4}$.

The first lemma is an obvious extension of [1, Lemma 16.1].

LEMMA 1. If $z=x+i y$, then

$$
|G(z)| \leqslant A(1+|z|) e^{\pi|y|}, \quad|G(z)| \geqslant B|y|(1+|z|)^{-2} e^{\pi|y|},
$$

and

$$
\left|G\left(\frac{1}{2}+i y\right)\right| \geqslant C
$$

where $A, B$, and $C$ are absolute positive constants (independent of the choice of $\lambda_{n}$ ).

For the remaining three lemmas we shall suppose that the $\lambda_{n}$ are given by (1).

LEMMA 2. $|G(x)|=O(1 / \sqrt{x})$ as $x \rightarrow \infty$.

Proof. We begin by observing that

$$
\frac{G(z)}{z}=\frac{\Gamma^{2}(5 / 4)}{\Gamma(5 / 4+z) \Gamma(5 / 4-z)} .
$$


Indeed, the right side-call it $H(z)$-is an entire function of order 1 , equal to 0 when $z= \pm \lambda_{n}(n=1,2,3, \ldots)$, and equal to 1 when $z=0$. Therefore, by Hadamard's theorem,

$$
\frac{G(z)}{z}=e^{A z} H(z)
$$

Since $G(z) / z$ and $H(z)$ are both even, $A=0$ and the assertion follows.

Using the identity

$$
\Gamma(z) \Gamma(1-z)=\frac{\pi}{\sin \pi z},
$$

we obtain

$$
\frac{G(z)}{z}=c \frac{\sin \pi(z-1 / 4)}{\Gamma(z+5 / 4) / \Gamma(z-1 / 4)}
$$

where $c=\Gamma^{2}\left(\frac{5}{4}\right) / \pi$. Using Stirling's formula

$$
\Gamma(x+1) \sim(2 \pi)^{1 / 2} x^{x+1 / 2} e^{-x} \quad(\text { as } x \rightarrow \infty),
$$

we find that

$$
\frac{\Gamma(x+5 / 4)}{\Gamma(x-1 / 4)} \sim x^{3 / 2}
$$

and so

$$
|G(x)| \sim c \frac{|\sin \pi(x-1 / 4)|}{\sqrt{x}} \quad(\text { as } x \rightarrow \infty) .
$$

This proves the lemma.

Lemma 3. As $n \rightarrow \infty$,

$$
\int_{-\infty}^{\infty}\left|\frac{G(x)}{x-\lambda_{n}}\right|^{2} d x=O(1 / n)
$$

and

$$
\int_{-\infty}^{(n+1) / 2}\left|\frac{G(x)}{x-\lambda_{n}}\right|^{2} d x=O\left(\frac{\log n}{n^{2}}\right) .
$$

Proof. It was shown in [2] that

$$
G(z)=\int_{-\pi}^{\pi} f(t) e^{i z t},
$$

where $f(t)$ is a multiple of $\left(\cos \frac{1}{2} t\right)^{-1 / 2} \sin \frac{1}{2} t$. Integration by parts then yields

$$
\frac{G(z)}{z-\lambda_{n}}=\int_{-\pi}^{\pi} g_{n}(t) e^{i z t} d t
$$

where

$$
g_{n}(t)=-i e^{-i \lambda_{n} t} \int_{-\pi}^{t} f(x) e^{i \lambda_{n} x} d x
$$


Since $f$ is integrable over $(-\pi, \pi)$, it follows that every $g_{n}$ belongs to $L^{2}(-\pi, \pi)$. Applying Plancherel's formula to (5) we find that

$$
\int_{-\infty}^{\infty}\left|\frac{G(x)}{x-\lambda_{n}}\right|^{2} d x=\frac{1}{2 \pi} \int_{-\pi}^{\pi}\left|g_{n}(t)\right|^{2} d t .
$$

But the right side is $O(1 / n)$ as $n \rightarrow \infty$ [2], and this establishes (3).

To prove (4) let us write

$$
\int_{-\infty}^{(n+1) / 2}\left|\frac{G(x)}{x-\lambda_{n}}\right|^{2} d x=\left(\int_{-\infty}^{-1}+\int_{-1}^{1}+\int_{1}^{(n+1) / 2}\right)\left|\frac{G(x)}{x-\lambda_{n}}\right|^{2} d x=I_{1}+I_{2}+I_{3} .
$$

Clearly, $I_{2}=O\left(1 / n^{2}\right)$ as $n \rightarrow \infty$. By Lemma 2,

$$
\begin{aligned}
I_{3} & =O\left(\int_{1}^{(n+1) / 2} \frac{1}{x\left(x-\lambda_{n}\right)^{2}} d x\right) \\
& =O\left(\frac{1}{\lambda_{n}^{2}} \int_{1}^{(n+1) / 2} \frac{1}{x} d x\right)=O\left(\frac{\log n}{n^{2}}\right) .
\end{aligned}
$$

Similarly, since $G(z)$ is odd,

$$
I_{1}=O\left(\int_{-\infty}^{-1} \frac{1}{-x\left(x-\lambda_{n}\right)^{2}} d x\right)=O\left(\int_{1}^{x} \frac{1}{u\left(u+\lambda_{n}\right)^{2}} d u\right)
$$

Using

$$
\frac{1}{u\left(u+\lambda_{n}\right)^{2}}=\frac{1}{\lambda_{n}^{2}}\left(\frac{1}{u}-\frac{1}{u+\lambda_{n}}\right)-\frac{1}{\lambda_{n}} \frac{1}{\left(u+\lambda_{n}\right)^{2}}
$$

we find that

$$
\int_{1}^{\infty} \frac{1}{u\left(u+\lambda_{n}\right)^{2}} d u=\frac{1}{\lambda_{n}^{2}} \log \left(1+\lambda_{n}\right)-\frac{1}{\lambda_{n}} \frac{1}{1+\lambda_{n}}
$$

and hence

$$
I_{1}=O\left(\frac{\log n}{n^{2}}\right)
$$

This completes the proof.

We recall that two sequences $\left\{f_{n}\right\}$ and $\left\{g_{n}\right\}$ in $L^{2}(-\pi, \pi)$ are said to be biorthogonal if

$$
\int_{-\pi}^{\pi} f_{n}(x) g_{m}(x) d x=\delta_{m n} .
$$

LEMMA 4. The functions $\left\{h_{n}(x)\right\}$ defined by

$$
h_{n}(x)=\lim _{A \rightarrow \infty} \frac{1}{2 \pi} \int_{-A}^{A} \frac{G(u)}{G^{\prime}\left(\lambda_{n}\right)\left(u-\lambda_{n}\right)} e^{-i u x} d u
$$

form a sequence biorthogonal to $\left\{e^{i \lambda_{n} x}\right\}$ in $L^{2}(-\pi, \pi)$. The limit in the mean is of order 2. 
Proof. By virtue of (5), we have

$$
\frac{G(u)}{G^{\prime}\left(\lambda_{n}\right)\left(u-\lambda_{n}\right)}=\int_{-\pi}^{\pi} h_{n}(x) e^{i u x} d x
$$

where $h_{n}(x)=g_{n}(x) / G^{\prime}\left(\lambda_{n}\right)$ and $g_{n}$ is given by (6). Since $g_{n}$ belongs to $L^{2}(-\pi, \pi)$, so does $h_{n}$. But $G\left(\lambda_{m}\right)=0$ for every $\lambda_{m}$, and so

$$
\int_{-\pi}^{\pi} h_{m}(x) e^{i \lambda_{n} x} d x=\delta_{m n} .
$$

Thus the sequence $\left\{h_{n}(x)\right\}$ is biorthogonal to $\left\{e^{i \lambda_{n} x}\right\}$ in $L^{2}(-\pi, \pi)$, and (7) follows at once from the Fourier inversion formula.

3. Proof of the Theorem. We shall not reproduce Levinson's proof in its entirety. The basic ideas and their necessary modifications will be set forth explicitly, while those calculations that remain unchanged will simply be summarized.

Let $C$ denote a rectangular path, positively oriented, in the complex $\xi$ plane with vertices at $N+\frac{1}{2}+i M,-N-\frac{1}{2}+i M,-N-\frac{1}{2}-i M$, and $N+\frac{1}{2}-i M$, where $M$ and $N$ are positive integers. Let $G(z)$ be defined as in (2).

Using residues and Lemma 4, Levinson shows that

$$
\begin{aligned}
& \sum_{-N}^{N} h_{n}(y) e^{i \lambda_{n} x}-\frac{\sin (N+1 / 2)(x-y)}{\pi(x-y)} \\
&=\lim _{A \rightarrow \infty} \frac{1}{4 \pi^{2} i} \int_{-A}^{A} G(u) e^{-i u y} d u \int_{C} \frac{e^{i \xi x}}{G(\xi)(u-\xi)} d \xi .
\end{aligned}
$$

It follows that if $f \in L^{2}(-\pi, \pi)$, then

$$
\begin{aligned}
& \sum_{-N}^{N} e^{i \lambda_{n} x} \int_{-\pi}^{\pi} f(y) h_{n}(y) d y-\frac{1}{\pi} \int_{-\pi}^{\pi} f(y) \frac{\sin (N+1 / 2)(x-y)}{x-y} d y \\
& =\frac{1}{4 \pi^{2} i} \int_{-\pi}^{\pi} f(y) d y \lim _{A \rightarrow \infty} \int_{-A}^{A} G(u) e^{-i u y} d u \int_{C} \frac{e^{i \xi x}}{G(\xi)(u-\xi)} d \xi
\end{aligned}
$$

If it can be shown that the right side of (8) tends to zero as $N \rightarrow \infty$, then by well-known results of Fourier series

$$
\lim _{N \rightarrow \infty} \sum_{-N}^{N}\left\{e^{i \lambda_{n} x} \int_{-\pi}^{\pi} f(y) h_{n}(y) d y-\frac{e^{i n x}}{2 \pi} \int_{-\pi}^{\pi} f(y) e^{-i n y} d y\right\}=0 .
$$

Let $I_{1}(x)$ denote the absolute value of that part of the right side of (8) for which $\xi$ varies over the upper horizontal side of the rectangle $C$. Then

$$
\begin{aligned}
I_{1}(x)=\mid \int_{-\pi}^{\pi} f(y) d y \lim _{A \rightarrow \infty} & \int_{-A}^{A} G(u) e^{-i u y} d u \\
& \cdot \int_{-N-1 / 2}^{N+1 / 2} \frac{e^{i \xi x-M x}}{G(\xi+i M)(u-\xi-i M)} d \xi \mid .
\end{aligned}
$$

Let $g(u) /(2 \pi)^{1 / 2}$ be the Fourier transform of $f(y)$. Appealing to Levinson to justify a change in the order of integration (which is valid because Lemma 2 guarantees that 
$\left.G(u) /(u+i)^{2} \in L(-\infty, \infty)\right)$, we find that

$$
I_{1}(x)=\left|\int_{-\infty}^{\infty} G(u) g(u) d u \int_{-N-1 / 2}^{N+1 / 2} \frac{e^{-M x+i \xi x}}{G(\xi+i M)(u-\xi-i M)} d \xi\right| .
$$

Clearly,

$$
\left|\frac{G(u)}{u-\xi-i M}\right| \leqslant C_{1} N\left|\frac{G(u)}{u}\right| \text { for } M>1 \text { and }|\xi|<2 N,
$$

and hence (9) becomes

$$
I_{1}(x) \leqslant C_{1} N e^{M|x|} \int_{-N-1 / 2}^{N+1 / 2} \frac{d \xi}{|G(\xi+i M)|} \int_{-\infty}^{\infty}\left|\frac{G(u) g(u)}{u}\right| d u .
$$

By Hölder's inequality,

$$
\int_{-\infty}^{\infty}\left|\frac{G(u) g(u)}{u}\right| d u \leqslant\left(\int_{-\infty}^{\infty}\left|\frac{G(u)}{u}\right|^{2} d u\right)^{1 / 2}\left(\int_{-\infty}^{\infty}|g(u)|^{2} d u\right)^{1 / 2}
$$

and therefore

$$
\int_{-\infty}^{\infty}\left|\frac{G(u) g(u)}{u}\right| d u \leqslant C_{2}\left(\int_{-\pi}^{\pi}|f(y)|^{2} d y\right)^{1 / 2}
$$

by Plancherel's formula. With this, (10) becomes

$$
I_{1}(x) \leqslant C_{3} N e^{M|x|} \int_{-N-1 / 2}^{N+1 / 2} \frac{d \xi}{|G(\xi+i M)|} .
$$

By Lemma 1,

$$
|G(\xi+i M)| \geqslant B M\left(M^{2}+N^{2}\right)^{-1} e^{\pi M} \text { for }|\xi| \leqslant N+1 / 2 .
$$

Using this in (11), we obtain

$$
I_{1}(x) \leqslant C_{4} N^{2}\left(M^{2}+N^{2}\right) e^{-M(\pi-|x|)} \text { for }|x|<\pi .
$$

Let $I_{2}(x)$ denote the absolute value of that part of the right side of (8) for which $\xi$ varies over the right vertical side of the rectangle $C$. Then

$$
\begin{aligned}
& I_{2}(x)=\mid \int_{-\pi}^{\pi} f(y) d y \lim _{A \rightarrow \infty} \int_{-A}^{A} G(u) e^{-i u y} d u \\
& \quad \cdot \int_{-M}^{M} \frac{e^{-x \eta}}{G(N+1 / 2+i \eta)(u-N-1 / 2-i \eta)} d \eta \mid .
\end{aligned}
$$

Once again, interchanging the order of integration can be justified, and we find

$$
I_{2}(x) \leqslant\left|\int_{-\infty}^{\infty} G(u) g(u) d u \int_{-M}^{M} \frac{e^{-x \eta}}{G(N+1 / 2+i \eta)(u-N-1 / 2-i \eta)} d \eta\right| .
$$

With an obvious change of variable,

$$
I_{2}(x) \leqslant\left|\int_{-\infty}^{\infty} g(u+N+1 / 2) d u \int_{-M}^{M} \frac{G(u+N+1 / 2) e^{-x \eta}}{G(i \eta+N+1 / 2)(u-i \eta)} d \eta\right| .
$$


Let $\mu_{n}=-N+\lambda_{N+n}$. Then $\left|\mu_{n}-n\right| \leqslant \frac{1}{4}$ and

$$
\left|\frac{G(u+N+1 / 2)}{G(i \eta+N+1 / 2)}\right|=\left(\frac{u+1 / 2-\mu_{0}}{i \eta+1 / 2-\mu_{0}}\right) \prod_{1}^{\infty}\left(\frac{u+1 / 2-\mu_{n}}{i \eta+1 / 2-\mu_{n}}\right)\left(\frac{u+1 / 2-\mu_{-n}}{i \eta+1 / 2-\mu_{-n}}\right) .
$$

Let

$$
G_{N}(u)=\left(u-\mu_{0}\right) \prod_{1}^{\infty}\left(1-\frac{u}{\mu_{n}}\right)\left(1-\frac{u}{\mu_{-n}}\right)
$$

Using the two equations above, together with Lemma 1, Levinson goes on to show that

$$
\begin{aligned}
I_{2}(x) \leqslant & \frac{C_{5}}{(\pi-|x|)^{2}}\left(\int_{-\infty}^{-N / 2}\left|\frac{G_{N}(u+1 / 2)}{u+1 / 2-\mu_{0}}\right|^{2} d u\right)^{1 / 2}\left(\int_{-\infty}^{\infty}|g(u)|^{2} d u\right)^{1 / 2} \\
& +\frac{C_{6}}{(\pi-|x|)^{2}}\left(\int_{N / 2}^{\infty}|g(u+1 / 2)|^{2} d u\right)^{1 / 2}\left(\int_{-\infty}^{\infty}\left|\frac{G_{N}(u+1 / 2)}{u+1 / 2-\mu_{0}}\right|^{2}\right)^{1 / 2} \\
& +C_{7}\left(\int_{N / 2}^{\infty}|g(u+1 / 2)|^{2} d u\right)^{1 / 2} .
\end{aligned}
$$

In general, there is no simple majorant for $G_{N}(u)$ that is sufficiently close to $G_{N}(u)$ in magnitude to give a satisfactory appraisal of its size. However, when the $\lambda_{n}$ are given by (1), then $G_{N}(u)$ can be expressed explicitly in terms of $G(u)$. Observe to begin with that

$$
\begin{aligned}
\prod_{1}^{\infty}\left(1-\frac{u}{\mu_{n}}\right)\left(1-\frac{u}{\mu_{-n}}\right) & =\left(\frac{1-u / \mu_{-N}}{1-u / \mu_{0}}\right) \prod_{n=1}^{\infty}\left(1-\frac{u}{\mu_{-N+n}}\right)\left(1-\frac{u}{\mu_{-N-n}}\right) \\
& =\left(\frac{1-u / \mu_{-N}}{1-u / \mu_{0}}\right) \prod_{n=1}^{\infty}\left(1-\frac{u}{\lambda_{n}-N}\right)\left(1-\frac{u}{-\lambda_{n}-N}\right) .
\end{aligned}
$$

Since $\mu_{-N}=-N, \mu_{0}=\frac{1}{4}$, and, for $n \neq 0$,

$$
1-\frac{u}{\lambda_{n}-N}=\frac{1-(N+u) / \lambda_{n}}{1-N / \lambda_{n}}
$$

we obtain

$$
\prod_{1}^{\infty}\left(1-\frac{u}{\mu_{n}}\right)\left(1-\frac{u}{\mu_{-n}}\right)=\frac{1+u / N}{1-4 u} \cdot \frac{H(u+N)}{H(N)}
$$

where $H(u)=G(u) / u$. Thus,

$$
G_{N}(u)=\frac{(u-1 / 4)(1+u / N) H(u+N)}{(1-4 u) H(N)}=-\frac{1}{4} \frac{G(u+N)}{G(N)} .
$$


We can now estimate $I_{2}(x)$. First,

$$
\begin{aligned}
\int_{-\infty}^{\infty}\left|\frac{G_{N}(u+1 / 2)}{u+1 / 2-\mu_{0}}\right|^{2} d u & =\frac{1}{16|G(N)|^{2}} \int_{-\infty}^{\infty}\left|\frac{G(u+N+1 / 2)}{u+1 / 4}\right|^{2} d u \\
& =\frac{1}{16|G(N)|^{2}} \int_{-\infty}^{\infty}\left|\frac{G(x)}{x-\lambda_{N}}\right|^{2} d x
\end{aligned}
$$

Now, the proof of Lemma 2 showed that

$$
|G(x)| \sim C_{8} \frac{|\sin \pi(x-1 / 4)|}{\sqrt{x}}, \quad \text { as } x \rightarrow \infty,
$$

and hence

$$
|G(N)| \sim C_{9} / \sqrt{N}, \quad \text { as } N \rightarrow \infty .
$$

This, together with (3), shows that the integrals

$$
\int_{-\infty}^{\infty}\left|\frac{G_{N}(u+1 / 2)}{u+1 / 2-\mu_{0}}\right|^{2} d u
$$

remain bounded as $N \rightarrow \infty$.

In similar fashion, we see that

$$
\int_{-\infty}^{-N / 2}\left|\frac{G_{N}(u+1 / 2)}{u+1 / 2-\mu_{0}}\right|^{2} d u \sim \frac{C_{10}}{N} \int_{-\infty}^{(N+1) / 2}\left|\frac{G(x)}{x-\lambda_{N}}\right|^{2} d x .
$$

This, together with (4), shows that

$$
\int_{-\infty}^{-N / 2}\left|\frac{G_{N}(u+1 / 2)}{u+1 / 2-\mu_{0}}\right|^{2} d u \rightarrow 0, \quad \text { as } N \rightarrow \infty
$$

Finally, since $g \in L^{2}$,

$$
\int_{N / 2}^{\infty}|g(u+1 / 2)|^{2} d u \rightarrow 0, \quad \text { as } N \rightarrow \infty .
$$

Using these results in (13), we obtain

$$
\lim _{N \rightarrow \infty} I_{2}(x)=0, \quad|x| \leqslant \pi-\delta, \delta>0,
$$

uniformly in $x$. This holds entirely independently of $M$.

We denote by $I_{3}(x)$ the absolute value of that part of the right side of (8) for which $\xi$ varies over the lower horizontal side of the rectangle $C$. Clearly, inequality (12) remains valid when $I_{1}(x)$ is replaced by $I_{3}(x)$. We introduce $I_{4}(x)$ similarly related to $I_{2}(x)$.

The proof of the theorem is at hand. By virtue of (14), we can make $I_{2}(x)$, and therefore $I_{2}(x)+I_{4}(x)$, arbitrarily small by choosing $N$ sufficiently large. Fixing $N$ in this way, we can then make $I_{1}(x)+I_{3}(x)$ arbitrarily small by choosing $M$ sufficiently large, as is evident from (12). This shows that the right side of (8) approaches zero as $N \rightarrow \infty$. 
Thus, each function $f \in L^{2}(-\pi, \pi)$ has a nonharmonic expansion

$$
f(t) \sim \sum_{-\infty}^{\infty} c_{n} e^{i \lambda_{n} t},
$$

which is equiconvergent with its ordinary Fourier series, uniformly on each closed subinterval of $(-\pi, \pi)$. Since the system $\left\{e^{i \lambda_{n} t}\right\}$ is complete, its biorthogonal system $\left\{h_{n}(t)\right\}$ is uniquely determined, and hence the representation (15) is unique. This completes the proof.

\section{REFERENCES}

1. N. Levinson, Gap and density theorems, Amer. Math. Soc. Colloq. Publ., vol. 26, Amer. Math. Soc., Providence, R.I., 1940.

2. R. M. Redheffer and R. M. Young, Completeness and basis properties of complex exponentials, Trans. Amer. Math. Soc. 277 (1983), 93-111.

3. R. M. Young, An introduction to nonharmonic Fourier series, Academic Press, New York, 1980.

4. On complete biorthogonal syistenis, Proc. Amer. Math. Soc. 83 (1981), 537-540.

Department of Mathematics, Oberlin College, Oberlin, Ohio 44074 